Cahiers de recherches médiévales

Journal of medieval studies

$4 \mid 1997$

Être père à la fin du Moyen Âge

\title{
Les limites de l'autorité paternelle face aux droits patrimoniaux dans le Gévaudan médiéval (fin XIII ${ }^{\mathrm{e}}$ - fin $\mathrm{XV}^{\mathrm{e}}$ siècles)
}

Philippe Maurice

\author{
(2) OpenEdition \\ Journals \\ Édition électronique \\ URL : https://journals.openedition.org/crm/961 \\ DOI : $10.4000 / \mathrm{crm} .961$ \\ ISSN : 1955-2424 \\ Éditeur \\ Honoré Champion \\ Édition imprimée \\ Date de publication : 15 décembre 1997 \\ ISSN : 1272-9752
}

Référence électronique

Philippe Maurice, « Les limites de l'autorité paternelle face aux droits patrimoniaux dans le Gévaudan médiéval (fın XIIIe-fin XVe siècles) », Cahiers de recherches médiévales [En ligne], 4 | 1997, mis en ligne le 15 janvier 2007, consulté le 15 décembre 2022. URL : http://journals.openedition.org/crm/961 ; DOI : https://doi.org/10.4000/crm.961

Ce document a été généré automatiquement le 15 décembre 2022.

Tous droits réservés 


\title{
Les limites de l'autorité paternelle face aux droits patrimoniaux dans le Gévaudan médiéval (fin XIII ${ }^{\mathrm{e}}$-fin XV siècles)
}

\author{
Philippe Maurice
}

1 Sous l'Ancien Régime, l'autorité du père nous paraît souvent incontestable, peu éloignée de la patria potestas romaine ${ }^{1}$. Mais les juristes ont décelé une différence bien nette entre ces deux formes du pouvoir paternel en constatant que les droits du père romain ne cessaient qu'à la mort de ce dernier, alors que les coutumes des diverses régions de la France limitaient l'exercice du pouvoir du père, dans le temps, du fait de l'accès à la majorité de l'enfant ${ }^{2}$. Nos travaux sur le Gévaudan nous autorisent à aller plus loin et à affirmer que le pouvoir paternel était susceptible d'être contesté. Cependant, nous rappellerons que l'autorité d'un homme dépend de deux rapports de force, l'un est primitif et relève de l'ascendant personnel exercé par un individu, l'autre est social et découle du Droit ${ }^{3}$. La conjugaison de ces deux influences détermine le rôle et la place de chacun dans le cadre des relations humaines et, en l'occurrence, dans la cellule familiale. L'ascendant du père est une donnée subjective difficile à appréhender, sauf à l'aide de documents spécifiques, comme les lettres de rémission ou les chroniques par exemple. Une certitude s'impose, le pouvoir dépend généralement du sexe (masculin), de l'âge (le plus ancien) et du rang dans l'ordre généalogique (le père, voire le grand-père) qui déterminent tous un ascendant naturel et confèrent une aura de force, d'expérience et de sagesse. Le chef de famille est donc le père le plus âgé, ou «le vieux père »lorsqu'un aïeul vit encore ${ }^{4}$.

2 Dans cet article, notre réflexion s'étaiera principalement sur les sources notariales, afin de démontrer que les droits dont chaque membre de la famille jouit sur la possession $\mathrm{du}$ patrimoine permettent une redistribution des influences lors des décisions prises dans l'oustau ${ }^{5}$, remettant occasionnellement en question l'ordre primitif. En outre, elle repose sur l'exemple du Gévaudan de la fin du XIII ${ }^{\mathrm{e}}$ siècle à la fin du XV $\mathrm{XV}^{\mathrm{e}}$ siècle ${ }^{6}$, livrant 
ainsi un exemple régional qui n'est pas forcément exportable dans les autres pays de l'ancienne France, même si nous le supposons adaptable à bien des régions du Midi et plus particulièrement du Languedoc dont il fait partie?

3 Nous venons de dire que l'autorité paternelle était limitée dans le temps et s'exerçait sur les enfants non majeurs, ce qui nous contraint à préciser à quoi correspondait la majorité. En Gévaudan, comme en Forez, existe une double majorité. La première survient à la puberté qui se situe aux alentours de la quatorzième année. A cet âge, l'orphelin se libère généralement de son tuteur qui l'administrait entièrement afin de prendre un curateur qui le conseillera et l'autorisera à passer des contrats ; en somme, le pubère jouit du pouvoir d'agir personnellement avec le conseil de son curateur. Ensuite, à vingt-cinq ans, l'enfant devient majeur d'âge et effectivement capable en toute chose ${ }^{8}$. Cependant, deux choix de vie remettent en cause cette norme, la situation du fils de famille et l'émancipation. En effet, l'homme marié qui vit sous le toit paternel, autrement dit le «fils de famille», demeure soumis aux commandements du père, quand bien même il a lui-même des enfants, et cela jusqu'au décès de l'auteur de ses jours. Au contraire, le fils émancipé, même âgé de moins de vingt-cinq ans et vivant près de son père, devient capable et majeur ${ }^{9}$. Enfin, quelques exceptions classiques autorisent la remise en cause du droit que le père a de diriger ses fils : la folie et la prodigalité ${ }^{10}$.

4 De plus, à plusieurs reprises, nous rencontrons des fils qui, du vivant de leur père, sont placés sous la curatelle d'un tiers. Le 6 février 1318 (ns), de conserve avec ses grandpère, père, mère et femme, Bernard Chapelle vend des cens à un prêtre. Or, Bernard agit avec l'autorisation de son père, lui-même autorisé par le grand-père, et en compagnie de son curateur Jehan Chaste. De même, avant de transiger avec les prêtres du collège Bonconseil de Mende, en août 1372, dom Aldebert de Peyre, seigneur de Marchastel et chanoine de Mende, se déclare mineur de vingt-cinq ans et requiert l'official de Mende de lui donner dom Jehan Bonenfant, un licencié dans les deux droits, comme curateur ${ }^{11}$. Pourtant le père $d^{\prime}$ Aldebert, Astorg, baron de Peyre, est toujours en $v^{12}{ }^{12}$. Dans les deux cas, le fils en question est toujours mineur, Bernard Chapelle, en qualité de fils de famille, et Aldebert de Peyre comme mineur d'âge, mais les deux hommes ont déjà pris contact avec la vie active, le premier est marié et le second est chanoine de Mende. En outre, tous deux jouissent de droits patrimoniaux. Bernard est au moins maître des biens dotaux de sa femme et il a sans doute reçu, à l'occasion de son mariage, une part des biens de ses père et grand-père comme l'usage le recommande. Aldebert de Peyre tient son fief de Marchastel ${ }^{13}$. Naturellement placés sous l'autorité de leur père, ces garçons non émancipés se distancient de leur père en obtenant un curateur. Si nous ignorons tout de la procédure suivie par Bernard Chapelle, nous savons qu'Aldebert sollicite personnellement de l'official de Mende de le pourvoir d'un curateur, ce qui ne signifie aucunement qu'il y ait rupture entre le père et le fils puisqu'en 1376 Aldebert transige avec la communauté des prêtres de la cathédrale de Mende, à propos des droits féodaux de sa baronnie de Marchastel, en compagnie de son père, Astorg de Peyre ${ }^{14}$. En demandant un curateur, les deux fils de famille revendiquent donc leur indépendance, face à leur père, grâce à leur patrimoine. Ce curateur n'exerce pas l'autorité paternelle ${ }^{15}$, mais il intervient comme conseiller en matière foncière.

5 Notre réflexion se fonde sur une certitude : la définition de l'autorité exercée sur un enfant devient nécessaire face à la possession et à l'administration de biens. Pour 
preuve, en 1445, le bailli de Vébron est saisi d'une demande d'institution de curatelle et de tutelle en faveur des enfants Gras afin de permettre la constitution des dots des filles aînées. En effet, les orphelins sont gouvernés, depuis quelques années, par leur mère et leurs oncles paternels sans que nul ne se soit réellement posé de question mais, parvenues à l'âge du mariage, les filles doivent être dotées et il convient dès lors de fixer les règles permettant de décider en matière patrimoniale ${ }^{16}$. La jouissance des biens pose donc le problème de l'autorité, et la capacité de l'enfant s'affirme précisément en fonction des biens qu'il possède, que ce soit lorsque son père est mort, ou lorsqu'il vit. Le père jouit d'un pouvoir sur les personnes et sur les biens ${ }^{17}$, or nous allons démontrer que la capacité de l'enfant est source des limites de l'autorité paternelle. Aucune brèche ne s'ouvrirait dans cette dernière par le seul biais des rapports de force primitifs car le père, homme mûr, est fait pour commander, et la faiblesse du pouvoir paternel résulte des complexités du droit.

6 La relation entre possession patrimoniale et indépendance est telle que les actes d'émancipation du XIV ${ }^{e}$ siècle contiennent tous une donation ${ }^{18}$; chaque fois, devant le juge, le père déclare donner des biens au fils afin de lui permettre d'agir et pour le libérer de la patria potestas ${ }^{19}$. En novembre 1328, Jehan Meyssonier alloue un oustau à son fils Étienne ${ }^{20}$. Après avoir rappelé qu'Étienne est filius familiae, Jehan précise que son fils pourra «tester, codiciller, commercer, vendre, donner, ester en justice, et se défendre et agir, et porter plainte, et intenter et faire tous les autres actes légitimes ${ }^{21}$. Jehan mentionne bien que la cession de l'oustau permettra à son fils d'exercer pleinement son droit. De même, lorsqu'il se marie, en 1478, Guillaume Garin vit chez son père, il est fils de famille placé in potestate patris et son contrat de mariage ajoute même qu'il n'a aucun bien propre; en conséquence de quoi son père lui constitue une donatio propter nuptias représentant la moitié de ses avoirs afin de lui permettre d'entretenir son ménage ${ }^{22}$. L'idée avancée par ces émancipations est que la capacité juridique n'existe, ne se prouve, et n'est réellement attestée que si l'individu possède des biens et, par conséquent, que le fils de famille ne sort de l'autorité du père que s'il est possesseur d'un patrimoine.

7 Ces donations prouvent l'importance émancipatrice du patrimoine mais impliquentelles une fragilisation de l'autorité paternelle, ou plutôt un partage du pouvoir au sein de l'oustau? En Gévaudan, le départ de l'oustau libère l'enfant du pouvoir paternel, aussi ces émancipations et donations que nous venons d'évoquer ne sont-elles justifiées que dans la mesure où le fils demeure avec le père. C'est ainsi que plusieurs fils de la noblesse sont émancipés alors qu'ils étaient les héritiers en puissance du fief ${ }^{23}$. Dès lors, dans la maison, le fils jouit juridiquement de ses droits d'action dans les seules limites de ses titres de possession.

8 Cette liberté du fils résulte alors de la seule décision du père ; c'est en effet ce dernier qui décide d'associer son fils en lui conférant une partie de la capacité sur ses biens, et par conséquent sur la gestion du domaine et sur le droit de décider. Mais le Gévaudan est un pays où l'esprit communautaire est largement développé. Souvent, l'héritier potentiel reçoit donation d'un patrimoine dont les parents en vie conservent l'usufruit et la domination ${ }^{24}$. En contrepartie de cette avance sur la succession, le donataire travaille et vit avec les donateurs; si ces derniers sont trop vieux, en particulier si la mère est la seule survivante, il se charge de les nourrir et de pourvoir à leur entretien. Ainsi le système communautaire permet-il au fils d'intervenir dans la gestion familiale $^{25}$, et le père peut difficilement échapper à cette tendance coutumière qui 
consiste à s'associer un enfant. Dans les faits, nous avons souvent eu la confirmation de la participation du fils, héritier potentiel, pour vendre des biens, pour délivrer des quittances et des procurations, voire pour s'obliger ${ }^{26}$.

9 Toutefois, l'enfant, garçon ou fille, et pas seulement le futur héritier de l'oustau paternel, gagne également sa liberté lorsqu'il dispose de biens adventifs. En effet, s'il hérite d'un tiers, un parrain, un cousin, ou un oncle, par exemple, il jouit des droits de possession sur ces biens propres. Tant qu'il est mineur, son père gère ces derniers en qualité de «légitime administrateur». En fait, si le père gouverne naturellement ses enfants, il n'est que leur procureur en matière d'administration mobilière et immobilière ${ }^{27}$. Dès que les enfants parviennent à l'âge de raison, et même s'ils demeurent encore sous le toit familial, ils peuvent prétendre défendre leurs droits et, en tout cas, décider d'en jouir. Cependant, cette situation ne remet pas réellement en cause la place du père. Jusqu'à présent, nous notons que l'enfant dispose d'un certain libre arbitre dès lors qu'il possède des biens, sans avoir réellement la capacité de se dresser contre son père. Bien sûr, le rapport de force, toujours favorable au père, offre quelque influence à l'enfant; s'il a des immeubles inclus dans le patrimoine parental, il peut exercer un certain chantage, refuser de vendre lorsque le père désire réunir du numéraire, ou vendre une terre que son père souhaiterait affermer, voire refuser de cautionner son père. Ayant des titres patrimoniaux, il peut donc pratiquer une résistance passive.

D'ailleurs, à plusieurs reprises, les actions du père sont soumises à la ratification du fils et nous voyons Jehan Manas s'engager, en 1322, à faire ratifier par ses deux fils la constitution dotale réalisée au profit de sa fille Dolsette ${ }^{28}$. Dans tous les cas, nous sommes en présence d'adultes vivant en compagnie du père. Ainsi, en 1318, le chevalier Raymond de Mayrières promet-il de faire ratifier par son fils émancipé la cession d'une rente féodale qu'il consent ${ }^{29}$. Le majeur est donc logiquement appelé à confirmer les transactions immobilières de son père, mais les mineurs également. En 1340, Blavet de La Garde, majeur de quinze ans, mais mineur d'âge, autorisé par son père et sur mandat de ses parents, ratifie la vente d'un fief que ces derniers ont faite la veille ${ }^{30}$. Cependant, un pelletier mendois, Jehan Charcos, rappelle que son fils Étienne ne confirmera sa vente d'une maison qu'une fois qu'il sera parvenu à l'âge de quatorze ans ${ }^{31}$, seuil de la puberté. Il est évident que cette participation ne tend pas à faire valoir les droits de l'enfant, mais plutôt à défendre ceux de l'acheteur puisque l'enfant devenu un adulte, en pleine maitrise de ses droits, est toujours susceptible de requérir sa restitutio in integrum, soit l'annulation des actes passés sous sa minorité. Toutefois, le fils jouit alors réellement d'un moyen de pression sur le père. Une parade existe pour protéger l'acquéreur et elle devient également une arme contre le fils, celle de l'amende infligée au père en cas de non-ratification. Ainsi Raymond de Mayrières accepte-t-il de se soumettre à une amende de dix livres si la ratification de son fils n'est pas enregistrée, or les dix livres payées par le père seront perdues par le fils, sur son hoirie ${ }^{32}$.

Si les actes d'obstruction, ou de revendication, du garçon deviennent plus pressants, plus énergiques, et que le père soit un homme vindicatif, l'enfant en paiera les conséquences. En 1458, Jehan Atger «Fresquet» se querelle avec son père, Pierre, qui lui avait promis un tiers de ses biens tout en en retenant l'usufruit. Les deux hommes finissent par transiger et Pierre cède divers immeubles, mais la vengeance se concrétise quelques années plus tard. Jehan est déshérité par sa mère qui lui préfère une nièce, fille de la sœur de Jehan, en 1464, et par ledit Pierre qui transmet ses héritages à 
François Atger «Fresquet», fils dudit Jehan, en $1467^{33}$. Dans les pays de droit écrit, l'exhérédation est l'arme la plus redoutable et la plus efficace dont dispose le père, ou la mère, pour se faire obéir. Ce simple fait conforte l'idée que le pouvoir émane en partie des droits patrimoniaux; le père commande chez lui parce qu'il est le tenancier du patrimoine, et pour chaque membre de la maison une logique s'impose: pas de biens, pas de pouvoir.

Le père est généralement le possesseur du patrimoine, soit parce qu'il a hérité de son père et s'est installé sur le domaine familial, soit parce qu'il était un cadet exclu de la succession qui a réussi, par son travail, à se constituer un petit capital. Mais il peut aussi s'être fixé «en gendre ». L'installation uxorilocale n'est pas rare en Gévaudan, plus du quart des jeunes qui s'installent chez des parents le font matrilocalement, et un peu moins des trois quarts de ceux qui fondent une famille nucléaire demeurent sur un fonds dotal ${ }^{34}$. Dans ce cas, le père est avant tout le mari de la mère, et il vit sur les biens dotaux et paraphernaux de son épouse. Les droits de possession sont entre les mains de la femme même si le mari les administre. En Gévaudan, la femme ratifie les transactions que son époux réalise sur sa fortune, aliénations ou accroissements. Le mari doit s'obliger sur ses immeubles lorsqu'il touche à ceux de sa femme; or s'il vit chez elle, sa liberté, ses choix et son pouvoir sont indéniablement limités. Mais l'esprit du temps veut que l'homme commande, et nul ne songe réellement à porter atteinte à l'image virile du chef de l'oustau. Lors des conflits et des querelles de voisinage, c'est l'homme qui se dresse, vigoureux, prêt à se battre ; à l'occasion des élections des représentants de la communauté d'habitants, consuls, syndics ou simples procureurs, c'est l'homme qui participe au vote dont les femmes sont exclues. Tant que sa femme vit, ce mari-père n'a rien à redouter puisqu'il est le «seigneur et usufruitier» de la dot, mais la mort ébranle cette apparente sécurité.

13 En effet, juridiquement, le mari est le maître de la dot tant que la femme vit ; au décès de cette dernière, la dot revient aux constituants si l'union est demeurée stérile ou aux enfants légitimes s'il y en a. Puisque nous nous intéressons à l'autorité du père, il est bien évident que seule la seconde perspective retiendra notre attention. Sauf mesure contraire, au décès de la femme, les héritages passent logiquement entre les mains des enfants, successeurs naturels des biens dotaux et paraphernaux de leur mère, qui peuvent les gérer. Cette pratique rencontrée en Gévaudan n'offre rien d'exceptionnel et en Bordelais les coutumes accordent «aux enfants en puissance de père une pleine capacité sur les biens de leur mère prédécédée, et en particulier un libre pouvoir de disposition à condition qu'ils soient en âge $»^{35}$. Le père n'est plus qu'un veuf qui vit chez ses enfants et, si l'un de ses fils est déjà un homme d'âge mur, ce dernier est le chef potentiel! Le pouvoir naturel du père, que nous qualifions de primitif, se heurte alors à l'autorité juridique du fils qui découle du droit patrimonial. Tant que la bonne entente règne entre les habitants de l'oustau, tout va bien, mais au moindre conflit la lutte oppose l'ascendant et les règles de droit. Dans de tels cas de figure, le père ne dispose de guère plus de pouvoir que les veuves n'en ont en elles-mêmes.

La liberté de tester et de disposer des biens en vigueur dans le midi offre deux parades à ce problème, autant à l'avantage du veuf-père, qui nous préoccupe, qu'à celui de la veuve. La première repose sur la désignation de l'héritier universel. Si la maîtresse du domaine teste en faveur de son époux, ce dernier deviendra le possesseur légal, au détriment des enfants. C'est le choix fait par $15 \%$ des femmes qui ne choisissent qu'un héritier universel, toutefois $64 \%$ appellent un descendant ${ }^{36}$. Le veuf-héritier sera alors 
généralement chargé d'un fidéicommis en faveur de ses enfants. Le second recours consiste à offrir au mari survivant l'usufruit des héritages légués à un enfant. Le père jouit donc viagèrement d'un usufruit sur les possessions héritées par l'enfant, mais son statut d'usufruitier est beaucoup plus incertain que celui d'héritier.

L'exemple du couple de Guillaume Pic et de Saure de Carnac illustre parfaitement la situation du conjoint-usufruitier. A l'origine, le 10 octobre 1436, Guillaume Pic, fils de Jacques, de Saint-Laurent-de-Trèves, épouse Saure, fille de Jehan de Carnac, des Ablatats (Fraissinet-de-Fourques), dans les Cévennes ${ }^{37}$. Jehan promet en dot, la moitié de ses biens au mariage, et le reste après sa mort, mais il conserve la toute puissance sur l'ensemble, pour lui et pour sa femme, avec la faculté de tester, tout en réservant les dots de ses autres enfants. Les jeunes mariés devront vivre avec les Carnac. Guillaume apportera dans l'oustau de son beau-père cinquante livres tournois de ses propres et dix livres allouées par son père. Le 26 octobre, Guillaume délivre quittance de ses droits successoraux à son frère, Pierre Pic, en échange des dix livres. Dès lors, Jehan de Carnac, son gendre, sa femme et sa fille résident et travaillent ensemble alors que le domaine des Pic est exploité par Jacques et Pierre Pic auxquels s'associe Pierre Pic junior, frère de Pierre et de Guillaume, en $1443^{38}$. Jehan de Carnac vit encore longtemps puisqu'il ne teste qu'à la fin de l'année 1468. Il adresse alors des legs à ses filles, Guillauma, Béatrice et Astruge, toutes mariées, et à son fils Gervais, dotés et exclus de la succession. Il confirme l'institution universelle en faveur de Saure ${ }^{39}$. Dans les mois qui suivent, Jehan disparait. Le 6 août 1470, son petit-fils, Anthoine Pic, se marie avec Jehanne Pin qui lui apporte trente-huit livres en plus du trousseau nuptial. A cette occasion, Guillaume Pic, en son nom propre et en celui de Saure, alloue à Anthoine les deux tiers de leurs patrimoines respectifs, retenant le dernier tiers, tout en déclarant conserver la puissance, l'usufruit et l'administration de l'ensemble. Les mêmes clauses qu'en 1436 sont reprises : dotation des autres enfants, droit de tester et obligation de vie commune. Le 12 septembre, Saure de Carnac ratifie la donation faite par son mari.

Guillaume et Saure doivent alors envisager l'éventuelle disparition de l'un d'entre eux et le veuvage de l'autre. Le 17 avril 1472, ils rédigent leurs testaments en faveur $\mathrm{d}^{\prime}$ Anthoine ${ }^{40}$. Leurs autres enfants, Jehan, Guillaume, Claude, Anthonie et Bérengère seront entretenus jusqu'à leur mariage et dotés, mais l'un des fils pourra suivre des études pour devenir prêtre. Saure bénéficie de la puissance, de la domination et de l'usufruit sur les biens de Guillaume, et ce dernier de la puissance et de l'usufruit sur ceux de Saure. Le survivant régira et gouvernera les enfants sans la moindre contradiction. Une clause précise que Guillaume jouira du victum et vestitum nécessaire. Ce dernier point marque bien une différence entre les deux époux. Saure, devenue veuve, vivra chez elle, alors que Guillaume, veuf, demeurera chez son fils et il convient de lui garantir son entretien, en plus des clauses juridiques classiques. Certes Guillaume se voit conférer un usufruit, mais en cas de violent conflit avec Anthoine sa position sera inconfortable! Anthoine sera le véritable possesseur de l'hoirie de Saure, il est associé à la gestion patrimoniale des deux tiers des biens familiaux depuis deux ans, et il doit nourrir ses frères et sœurs. Sa place, privilégiée depuis ses noces, deviendra prépondérante après la mort de sa mère même si elle est subordonnée à de pesantes charges familiales.

17 De quels droits disposera Guillaume face à son fils? Outre la clause d'usufruit et de puissance patrimoniale, il conserve éventuellement la propriété sur les soixante livres 
tournois apportées chez les Carnac en 1436. Cette somme s'avère sans doute la plus dissuasive menace contre une éventuelle rébellion d'Anthoine. En effet, en cas de rupture définitive entre le père et le fils, ce dernier devra restituer au moins vingt livres; en outre Guillaume pourra casser la donation des deux tiers de ses avoirs consentie en 1470 et contraindre Anthoine à lui reverser les soixante livres. Une telle restitution n'exonérerait pas Anthoine de l'obligation de nourrir et de doter ses frères et sœurs sur l'hoirie de Saure. Dans ce cas précis, il est clair que le père et le fils, après la mort de la mère, seront associés; bon gré, mal gré, ils vivront sur les terres qui furent à Jehan de Carnac et dont Anthoine aura hérité par le biais de sa mère. Les relations familiales reposeront sur l'ascendant naturel du père, sur la soumission $d u$ fils, et certainement sur la souplesse et la volonté à composer de l'un et de l'autre.

Qu'advient-il du père, veuf, originellement installé «en gendre », qui ne parvient pas à s'entendre avec son fils héritier universel des biens maternels ? La réponse est apportée par les clauses concernant la famille Bragose et elle correspond au sort réservé traditionnellement à la veuve installée chez un fils héritier du père : son éviction contre le paiement d'une pension et (ou) la garantie d'un logement ${ }^{41}$. En effet, le 8 avril 1473, Isabelle, fille et héritière universelle de Pierre André, de Villeneuve (Vébron), teste en faveur de ses deux fils, Guillaume et Anthoine. Elle assure à Pierre Bragose, son mari, le victum et vestitum à vie, ainsi que la capacité de tester jusqu'à la valeur de cinq livres. Toutefois, elle ajoute qu'au cas où la vie commune avec leurs enfants serait impossible Pierre recevrait un soutol pour son logement et, chaque année, quatre setiers de froment et d'orge, dix livres de fromage, vingt-cinq livres de porc salé, quatorze livres de laine, et vingt sous tournois. Elle précise encore qu'au cas où Pierre vivrait avec un seul de leurs fils, le second devrait payer la moitié de la pension ${ }^{42}$. Pourtant, Pierre Bragose avait naguère jouit d'une certaine attention des André puisque sa belle-mère, Saure, l'avait institué cohéritier universel avec sa femme, Isabelle, en $1445^{43}$. Cela démontre bien la fragile situation du père installé "en gendre ", et cela malgré les prévenances de la belle-famille. En fait, Pierre Bragose a tenté de conserver la maîtrise de l'oustau le plus longtemps possible. Le 25 janvier 1462, il a marié son fils, Guillaume, avec Bilone Pagès qui a apporté trente-trois livres et douze brebis en dot, sans rien lui céder. Huit ans plus tard, le 24 septembre 1470, c'est au tour d'Anthoine de convoler. La nouvelle épouse apporte un chasal, une châtaigneraie, une autre terre, quinze livres, mais Pierre Bragose n'alloue toujours rien à ses fils. Malgré cela, Guillaume et Anthoine décident de s'affrérer pour mener vie commune ${ }^{44}$. Le choix formulé par Isabelle André en 1473 est rationnel : Pierre Bragose est vieux, il réside sur les biens des André, leurs deux fils sont élus héritiers et ont fait pacte de vivre ensemble. Le mari-père ne dispose que des faibles droits légués par sa belle-mère, non de ceux des André; s'il devient veuf, il vivra chez ses deux fils qui sont explicitement désignés comme nouveaux chefs. Le droit naturel du père, son ascendant, sa force et sa sagesse sont sans doute considérés comme émoussés en raison de son âge et il ne dispose pas de droits de possession; le rapport de force est contre lui.

Le veuf naguère marié uxorilocalement dont les enfants recueillent l'universalité de l'hoirie de sa défunte femme se trouve donc dans une position inconfortable. Tant que ses enfants seront mineurs, l'homme agira avec une tranquille sérénité, simplement contraint de protéger les biens dont il aura la garde. Il peut même être nommé tuteur ${ }^{45}$, contre toute attente puisqu'il est théoriquement le légitime administrateur de ses 
enfants; il est clair que la veuve conforte ainsi son statut, et son autorité sur la patrimoine qui appartient aux enfants.

En conclusion, une question se pose. Nous orientons-nous vers une égalité père-fils ? Nous n'en avons jamais réellement trouvé la trace et nous devons seulement relever les indices découlant des droits ouverts par le patrimoine. Toutefois, dans le Sud-Ouest, au cœur du pays landais, Jacques Poumarède a découvert que les coutumes locales, fortement communautaires, faisaient du fils demeurant au même pain et au même vin l'égal du père ${ }^{46}$. En Gévaudan, le fils est normalement soumis au père, même au sein des familles souches réunissant un père à son fils marié et père d'enfants. Et il serait faux de penser que le père est couramment dans une position précaire; en général il est le chef incontestée ${ }^{47}$. Il ne faut donc pas tomber d'un extrême à l'autre, le fils est généralement soumis au père. Mais il est toutefois clair que le fils qui possède son propre patrimoine peut s'affirmer et défendre son indépendance, et que ce sont ses droits patrimoniaux qui lui offrent cette possibilité. De même, le fils qui détient tous les titres de possession sur le patrimoine et qui a atteint l'âge de la majorité légale de vingt-cinq ans peut expulser son père de la maison, comme le prévoient les Bragose, prouvant ainsi que l'autorité dépend des droits patrimoniaux.

\section{NOTES}

1. Après avoir rappelé que la situation était totalement différente entre les pays coutumiers, où l'autorité du père était moins absolue, et les pays de droit écrit dans lesquels la patria potestas romaine était observée, Jacques Poumarède nuance encore cette réalité en expliquant que c'est surtout à partir du XVI siècle que la doctrine savante imposa «cette application rigoriste des lois romaines »; « Les tribulations de l'autorité paternelle de l'ancien droit au code Napoléon ", Annales de l'université des sciences sociales de Toulouse, t. LXII, 1994, p. 17.

2. P. Ourliac et J.L. Gazzaniga, Histoire du droit privé français, de l'An mil au Code civil, Paris, Albin Michel, 1985, p. 275.

3. Lorsqu'il définit l'autorité et le pouvoir, le sociologue Guy Rocher, s'inspirant de Ralf Dahrendorf et de Max Weber, déclare que «Le pouvoir s'attache à la personne ; il peut dépendre de la force physique d'une personne, de son talent, d'un charisme, aussi bien que du poste qu'elle occupe. Par contre, l'autorité est strictement attachée au poste occupé ou au rôle rempli dans l'organisation sociale. " (Introduction à la sociologie générale, t. 3, Le changement social, Paris, HMH, 1968, éd. 1972, p. 111). Il n'est pas inintéressant de constater que le terme potestas exprime aussi bien le concept du pouvoir que celui de l'autorité, le premier découlant de l'ordre naturel et le second des règles sociales.

4. Encore devons-nous noter que la situation de chef de famille et l'exercice de l'autorité paternelle sont deux choses distinctes, ainsi le grand-père, chef de famille, n'exerce-t-il pas l'autorité paternelle sur son petit-fils du vivant de son propre fils. Plusieurs actes prouvent que le fils est sous l'autorité de son père, lui-même sous 
l'autorité du grand-père. Ainsi, le 7 février 1405 (ns), lors de son mariage, Guigot Gachon, d'Aumont, s'engage-t-il, sous l'autorité de son père, Bartholomé, à restituer la dot de sa future femme si celle-ci meurt avant lui sans enfant, alors que le même Bartholomé ne constitue une donatio propter nuptias au profit dudit Guigot que sous l'autorité de son propre père, Bertrand, grand-père de Guigot (Archives départementales de la Lozère, 3E.2666/ fol. 177).

5. Le terme «oustau » désigne aussi bien la maison en tant qu'immeuble («oustau » en occitan) que la maisonnée («oustalado » en occitan).

6. La période retenue, biséculaire, peut sembler vaste et la présentation d'actes distants de cent cinquante ans peu cohérente ; nous signalerons donc que la pratique demeure identique pendant les XIVe et $\mathrm{XV}^{\mathrm{e}}$ siècles; seules les limites de cette étude, et la nécessaire clarté du texte, nous empêchent de livrer les nombreux exemples illustrant une si longue période.

7. Le Gévaudan correspond au département de la Lozère ; il faisait et fait toujours partie du Languedoc. Il ressortit donc au droit écrit. Toutefois, comme dans beaucoup de pays du Midi, le droit savant y est souvent remis en question par la pratique notariale qui l'accommode en fonction des usages locaux.

8. Ph. Maurice, La famille en Gévaudan au XV siècle, d'après les sources notariales (1380-1483), thèse de Doctorat, Tours, 1995, pp. 580-602. A paraître aux P.U. de la Sorbonne. H. Richardot, «Tutelle, curatelle et émancipation des enfants légitimes en Forez au XIII siècle ", RHDFE, 1945, pp. 30-31.

9. Le décret d'émancipation le plus ancien que nous ayons retrouvé fut prononcé par le juge du chapitre de Mende en 1308, en faveur de Jehan Vaycière, d'Almondès (ADL, 319B1, fol. 56). Toutefois un acte de 1291 rapporte que, quelques années auparavant, Bernard Chabrier, citoyen de Mende, émancipa son fils (ADL, 3E.1588, fol. 189v).

10. Nous avons découvert de tels cas en Gévaudan, au XV siècle : père aliéné déchu de ses droits et placé sous curatelle alors que ses enfants recevaient des tuteurs et curateurs, mais aussi père prodigue dont le fils fut placé sous le gouvernement et la tutelle d'un beau-frère (oncle de l'enfant). Ces décisions furent bien entendu prises par des juges (Ph. Maurice, op. cit. pp. 542-548).

11. Chapelle (ADL, G.1351, fol. 77v), Peyre (ADL, 3E.1430, fol. 37v).

12. Bien des années auparavant, en 1329, un autre Aldebert de Peyre, également seigneur de Marchastel, agissait pour sa part sous l'administration de son propre père, Astorg, également baron de Peyre (ADL, 3E.1430, fol. 120, 128).

13. Le fief de Marchastel constituait l'apanage perpétuel des cadets de Peyre voués à l'Église et prénommés Aldebert ; cet Aldebert tenait donc naturellement le fief destiné à l'établir au sein de l'Église.

14. ADL, G.1356, fol. 114.

15. Puisque Bernard Chapelle agit avec l'autorisation de son père.

16. ADL, 3E.2027, fol. 140.

17. Il ne faut pas oublier que le père est également un époux et que son autorité de père se double de celle d'un mari, même si cette seconde face de son autorité ne s'intègre pas à l'objet de notre exposé.

18. Vers la même époque, en Rouergue, Anne-Marie Landès-Mallet note que l'allocation d'une libéralité à l'occasion des émancipations est presque générale (La famille en Rouergue au Moyen Âge. Étude de la pratique notariale, Rouen, P.U. de Rouen, 1985, p. 123). 19. Le terme apparaît bien dans plusieurs actes. 
20. De même, en 1343, Pierre Bonfils cède le manse de Chasul (ADL, 1441, fol. 40v). Cette pratique remonte au moins au XIII ${ }^{\mathrm{e}}$ siècle puisque Bernard Chabrier attribua à son fils le manse de Costa Anoles entre 1270 et 1291 (un manse correspond à un lieu-dit). 21. Posset et valeat testare, codicillare, mercare, vendere, donare, stare in judicio, et se deffendere, agireque, et experiri et aliter omnes alios actus legitimus facere excere (ADL, 3E. 1430 , fol. $74 \mathrm{v})$.

22. ADL, 3E.718, fol. 240v, 242.

23. Ph. Maurice, op. cit. pp. 542-548.

24. Ph. Maurice, op. cit. pp. 452-457.

25. Fréquemment, le père, la mère et le fils instrumentent ensemble.

26. Ibid, pp. 554-559.

27. Une reconnaissance féodale faite en 1443 par un habitant de Chirac prouve que le père agit alors comme procureur général de ses enfants : reconnaissance de Jacques Cuoc (ADL, 3E.1563, fol. 37 et ss).

28. ADL, 3E.1432, fol. 51.

29. ADL, G.1352, fol. $85 \mathrm{v}$.

30. ADL, G.1353, fol. 8.

31. En 1301, ADL, G.1350, fol. 32.

32. $\mathrm{Au} \mathrm{XV} \mathrm{X}^{\mathrm{e}}$ siècle, dix livres correspondent au montant d'une pension annuelle courante, ou à un titre clérical, or ce dernier descend souvent à cinq livres au cours de la première moitié du XIV ${ }^{\mathrm{e}}$ siècle. De même, plusieurs maisons valent un tel prix. En conséquence, cette somme est loin d'être symbolique et elle s'avère réellement pénalisante.

33. ADL, 3E.2889, fol. 210v, 2892, fol. 152v, 2894, fol. 155.

34. Nous devons toutefois préciser que le domicile de $80 \%$ des couples à structure nucléaire demeure inconnu, ces trois quarts se rapportent aux $20 \%$ connus.

35. J. Poumarède, «Puissance paternelle et esprit communautaire dans les coutumes du Sud-Ouest de la France au Moyen Âge ", Recueil de mémoire et travaux, Société d'histoire du droit, Montpellier, fasc. IX, 1974, p. 659.

36. $81 \%$ des testaments mentionnent l'usage de l'élection d'un héritier unique. Ph. Maurice, op. cit. pp. 316-326.

37. ADL, 3E.2014, fol. 79, 81. Les deux lieux sont distants d'une douzaine de kilomètres.

38. ADL, 3E.2017, fol. 60 .

39. ADL, 3E.2044, fol. 3v.

40. ADL, 3E.2044, fol. 24, 25.

41. Encore certaines veuves n'obtiennent-elle que la restitution légale de leur dot avec un éventuel augment. Ainsi, après avoir envisagé que leurs veuves ne pourraient peutêtre pas vivre avec leurs héritiers, Pierre Salvanh, de la Fage-Saint-Julien, laisse une pension à sa veuve potentielle, le 5 novembre 1470, (ADL, 3E.1086, fol. 16), Pierre Bonet, de Rieutort-de-Randon, une pension et un logement à la sienne, le 21 février 1471 (ns), (3E.611, fol. 141), et Anthoine Gaus, de Mende, un augment de dot en numéraire, le 28 mai 1473 (3E.1086, fol. 65).

42. ADL, 3E.2044, fol. 37v.

43. ADL, 3E.2017, fol. 116.

44. L'affrèrement peut s'être réalisé sur les biens propres des deux fils, ou sur leurs biens «à venir », soit la succession maternelle (ADL, 3E.2037, fol. 7v, 2042, fol. 19v). 
45. Ph. Maurice, op. cit. pp. 554-558.

46. J. Poumarède, loc. cit. p. 661.

47. Ainsi Jehan Monbel, juge de la cour temporelle de Mende, se dit-il administrateur de son fils, Raymond, qui est déjà un juriste reconnu dans la cité (Ph. Maurice, op. cit. p. 554-558).

\section{AUTEUR}

\section{PHILIPPE MAURICE}

35 rue du général Moulin, 14034 Caen. 